\title{
Lidil
}

Revue de linguistique et de didactique des langues

\section{La saillance du procès dans les constructions passives non canoniques. Étude contrastive français-hongrois}

The Salience of the Event in Non-Canonical Passive Constructions. A FrenchHungarian Contrastive Study

\section{Anna Sőrés}

\section{OpenEdition}

\section{Journals}

Édition électronique

URL : https://journals.openedition.org/lidil/9523

DOI : $10.4000 /$ lidil.9523

ISSN : $1960-6052$

Éditeur

UGA Éditions/Université Grenoble Alpes

Édition imprimée

ISBN : 978-2-37747-315-1

ISSN : $1146-6480$

\section{Référence électronique}

Anna Sőrés, «La saillance du procès dans les constructions passives non canoniques. Étude contrastive français-hongrois », Lidil [En ligne], 64 | 2021, mis en ligne le 01 novembre 2021, consulté le 24 novembre 2021. URL : http://journals.openedition.org/lidil/9523 ; DOI : https://doi.org/10.4000/ lidil.9523

Ce document a été généré automatiquement le 24 novembre 2021.

(C) Lidil 


\section{La saillance du procès dans les constructions passives non canoniques. Étude contrastive français-hongrois}

The Salience of the Event in Non-Canonical Passive Constructions. A FrenchHungarian Contrastive Study

Anna Sőrés

\section{Introduction, problématique}

1 Plusieurs études récentes ont apporté des nouveautés dans la description et dans l'analyse du passif à l'oral en français (Hamma, 2017, 2019, 2020 ; Hamma et coll., 2017). En particulier, elles ont souligné l'importance de la distinction entre forme canonique et autres formes, non canoniques, du passif ainsi que le fait qu'à l'oral il y a très peu d'occurrences des phrases types proposées dans les grammaires, manuels scolaires et méthodes pour FLE. En même temps, ces recherches ont relancé les discussions sur la «saillance » et, par opposition, sur «l'occultation» de l'un des constituants de la phrase transitive et elles ont mis la lumière sur le concept de « saillance du procès » qui n'a pas encore reçu assez d'attention.

Dans cet article, nous adoptons une approche contrastive : les résultats de l'analyse du français oral sont confrontés avec les données d'une langue dans laquelle il n'existe pas de construction passive dans le sens traditionnel du terme. Il s'agit du hongrois qui servira de démonstration de structures syntaxiques très différentes correspondant à la même fonction discursive du passif. Notons encore que pour les locuteurs du hongrois, l'apprentissage du passif dans les langues indo-européennes modernes le plus souvent étudiées, telles que l'anglais, l'allemand ou le français est difficile, non seulement pour 
l'absence de forme passive ${ }^{1}$ mais aussi pour la description insatisfaisante qu'en produisent les grammaires traditionnelles.

3 L'analyse repose sur un corpus bilingue, notamment un ensemble d'énoncés traduits du français en hongrois ; les énoncés représentent une forme simplifiée des constructions relevées dans le corpus orléanais (<http://eslo.huma-num.fr>), exploitées dans Hamma (notamment dans 2017 et 2019).

4 Le but de notre étude est de corroborer et, à la lumière de la typologie et d'une approche contrastive, de nuancer la différence entre formes canoniques et non canoniques. S'agissant d'une étude contrastive, on part de la forme canonique du français, à savoir la structure « être + participe passé + complément d'agent ».

5 Le résultat visé par cette étude est une meilleure compréhension du rapport entre les différentes formes en français et en hongrois. L'analyse du corpus bilingue montre que les énoncés passifs canoniques peuvent être traduits en hongrois uniquement par des énoncés actifs dans lesquels l'encodage morphosyntaxique correspond à sujet-agent et objet-patient. L'objet direct peut être topicalisé et se placer en tête de phrase. En revanche, la plupart des formes non canoniques se rapprochent davantage de la voix moyenne que du passif, ce qui peut également être illustré par les énoncés hongrois. En fait, si le hongrois moderne n'utilise pas de passif, cette langue se caractérise plutôt par une opposition entre actif et moyen.

\section{Arrière-plan conceptuel : opérations sur la valence et détransitivisation}

\subsection{Passif}

Le passif est considéré comme un phénomène à l'intersection de la morphosyntaxe et de la pragmatique ${ }^{2}$. Il est possible et pratique de partir d'une définition syntaxique puisque, quelle que soit l'évaluation des énoncés du point de vue discursif, le dénominateur commun est la diminution du nombre d'arguments dans la phrase. La définition que nous empruntons à Creissels (2001) prend pour point de départ une phrase transitive :

[Le passif est la] modification morphologique du verbe liée à la «destitution» de

l'argument traité comme sujet dans la construction de base et à la " promotion » de

l'argument traité comme objet dans la construction de base du verbe,

traditionnellement désignée comme active.

Par conséquent, le passif est " canonique » si la destitution (ou la suppression) du sujet s'accompagne de la promotion de l'objet du verbe transitif, comme dans un exemple prototypique : La police a barré la route $\rightarrow$ La route a été barrée par la police. Bien connu dans les langues indo-européennes, ce type de passif n'est pas inconnu dans d'autres langues non plus (voir Creissels, 2006, II, p. 10, sur le peul). Dans certaines langues, l'objet indirect peut aussi être promu, comme en anglais Mary was given a book. D'autres constructions s'y rapprochent, comme le passif "oblique ", this bed has been slept in (Creissels, 2001). Certaines langues connaissent le passif impersonnel, c'est-à-dire la destitution de l'unique argument d'un verbe intransitif, comme en allemand es wurde getanzt, littéralement 'il a été dansé, on a dansé'. Ce type d'énoncé s'écarte évidemment du prototype dans la mesure où l'évènement ne nécessite qu'un unique participant ${ }^{3}$. 
8 D'autres linguistes s'approchent de la définition du côté pragmatique. Pour Shibatani (1985, p. 37) la fonction fondamentale du passif prototypique est la défocalisation de l'agent. Cette propriété peut être observée dans ses exemples en japonais dans lesquels le morphème spécifique du passif est utilisé dans la présentation d'évènements spontanés, non volontaires, ainsi que pour exprimer une certaine distance entre les interlocuteurs, comme c'est d'usage dans plusieurs langues de l'Asie du Sud-Est.

9 Ce qui est commun dans les phrases passives (Keenan \& Dryer, 2007, p. 341 ; Shibatani, 1985), c'est la nature affectée ${ }^{4}$ du sujet qui correspond à l'objet de la phrase active. C'est la propriété sur laquelle nous insisterons dans nos réflexions sur la distinction entre canonique et non canonique. En effet, la nature affectée du sujet est la propriété fondamentale des constructions de la voix moyenne à laquelle nous rapprocherons les formes non canoniques identifiées dans les exemples de notre corpus. À ce concept s'attache alors « la saillance du procès » dans la mesure où le procès sera mis en relief à la place des participants.

\subsection{Détransitivisation}

10 Puisqu'il s'agit d'opérations sur la valence, en particulier de celles qui permettent de diminuer le nombre des arguments de la phrase transitive, il faut se référer à l'article de Hopper et Thompson (1980) sur la notion de transitivité. Les auteurs proposent une conception scalaire de la transitivité, appréhendée comme un ensemble de paramètres impliquant non seulement le nombre de participants mais aussi leurs propriétés sémantiques ainsi que la nature de l'action (télique ou non, volontaire ou non) et le degré d'affectation de l'objet. Cette conception est particulièrement importante dans l'étude du passif parce que les phrases moins transitives se prêtent moins facilement à la passivation, ou bien elles prennent plus naturellement une forme non canonique. Quelques exemples simples de phrases transitives illustrent les considérations théoriques ci-dessus et en même temps renvoient à la difficulté d'un non-francophone qui apprend que la passivation est la propriété d'un verbe transitif.

\begin{tabular}{|c|}
\hline (1) \\
\hline $\begin{array}{l}\text { mon frère a construit une maison > La maison a été construite par mon frère ; } \\
\text { tu aimes Julie > ? Julie est aimée par toi; } \\
\text { j'ai oublié son nom > *son nom a été oublié par moi }\end{array}$ \\
\hline $\begin{array}{l}\text { les enfants ont mangé le gâteau > le gâteau a été mangé par les enfants } \\
\text { les enfants ont mangé du gâteau > ? du gâteau a été mangé par les enfants }\end{array}$ \\
\hline
\end{tabular}

11 On observe que l'utilisation de la forme canonique semble plus naturelle en cas de transformation d'une phrase en haut de l'échelle de la transitivité.

\subsection{Passif et moyen}

12 En utilisant la terminologie traditionnelle des grammaires du français ${ }^{5}$ et des langues romanes où on parle de « verbes pronominaux » ou de $s e$-verbes, il n'est pas évident de 
mettre en relation passif et moyen puisque ce dernier terme n'est généralement pas utilisé. Dans une étude contrastive impliquant le hongrois, en revanche, il est impossible de contourner le terme "moyen » (angl. middle), même s'il n'y a pas de véritable consensus sur la définition de ce phénomène en linguistique générale.

Le domaine du moyen a été défini en termes sémantiques, d'abord par Benveniste (1966, p. 173) qui affirme que «dans l'actif, les verbes dénotent un procès qui s'accomplit à partir du sujet et hors de lui. Dans le moyen, qui est la diathèse à définir par opposition, le verbe indique un procès dont le sujet est le siège ; le sujet est intérieur au procès ». D’après Lyons (1970, p. 286), « la voix moyenne, quand elle est en opposition avec la voix active, signifie que l'action ou l'état affecte le sujet du verbe ou ses intérêts ». Plus récemment, Kemmer (1993) a établi des sous-domaines sémantiques, tels que celui des verbes de changement d'état physique, des verbes d'émotion, etc.

Dans le présent cadre, il suffit de rappeler deux choses. Premièrement, le résultat d'une opération de type "moyen" offre différentes lectures, telles que réfléchie (il se lave), réciproque (ils se saluent), anticausative (il s'assied) et modale (ou facilitative, ou encore dispositionnelle: lavable). En contrastant les deux langues qui nous intéressent, on observera qu'en français, c'est la se-forme qui se prête à toutes ces interprétations, alors que le hongrois est riche en suffixes moyens permettant de faire certaines distinctions entre les lectures (Sőrés, 2003, 2006, 2013). Deuxièmement, lors d'une opération de type moyen, le sens du verbe reste inchangé, mais l'un des participants à l'évènement reste implicite : ce tissu se lave bien / est bien lavable ; la porte s'ouvre. Il s'agit bien de l'évènement 'laver' ou 'ouvrir', mais le participant qui apparaitrait dans une phrase active comme «agent » n'est pas ou ne pourrait pas être explicité, l'argument qui apparaitrait comme objet-patient est le seul explicité et son référent est affecté par l'évènement en question. La nature affectée du référent du sujet de la phrase de type moyen nous ramène vers la nature plus « saillante » de l'événement.

\subsection{Saillance et occultation}

Dans l'analyse du volet syntaxique du passif, nous avons opposé les termes de « promotion » et de « destitution ». Quant à l'équilibre entre les deux procédés, nous ne discutons pas si la passivation doit être analysée comme promotion de l'objet ou destitution du sujet. Du point de vue de l'énonciation, on utilise les termes «saillance » et «occultation " (Brahim, 2001), ce dernier correspondant à « défocalisation » chez Shibatani. Dans la plupart des exemples empruntés aux corpus oraux, c'est l'occultation de l'agent qui domine; dans d'autres, on observera que la saillance du procès exprimé par le verbe est plus marquée que l'occultation des constituants nominaux.

\section{Analyse du corpus}

\subsection{Le corpus et le regroupement des exemples}

16 Notre corpus se compose d'une cinquantaine d'énoncés du français oral, traduits en hongrois. Les énoncés sont empruntés à ESLO (Enquête sociolinguistique à Orléans) et ils sont présentés sous une forme abrégée, c'est-à-dire que l'on se sert des structures sans l'ensemble du contexte ou du paragraphe. Quoique l'ensemble soit un corpus 
bilingue, l'analyse ne sera pas une analyse contrastive complète dans la mesure où le corpus ne permet pas de faire des statistiques. L'objectif est de répertorier les constructions et de distinguer celles qui représentent le passif canonique et les noncanoniques. Ensuite, on soumettra les formes non canoniques dans les deux langues à une analyse plus détaillée.

Comme mentionné dans l'introduction, nous partons d'un énoncé passif avec complément d'agent. Ainsi, nous nous référons à la définition syntaxique citée (voir 2.1) qui prévoit la présence de l'expression des deux participants. Le premier ensemble de données inclut ainsi i) des phrases passives avec complément d'agent explicité, ii) des énoncés du corpus dans lesquels la présence du complément d'agent est nécessaire pour l'interprétation sémantique et iii) ceux dans lesquels l'expression de la cause constitue le deuxième argument.

Dans le deuxième ensemble de données seront examinés les énoncés de sens passif sans complément d'agent ou de cause explicité. Certains restent plus proches du prototype, vu leur forme en "être + participe passé ", mais ils illustrent la fonction discursive du passif, à savoir la « défocalisation » de l'agent ou la diminution de son importance. Dans ces exemples, on peut tester la possibilité d'ajouter un agent. Dans d'autres exemples en revanche, en particulier dans le cas des adjectifs en -ble et des formes verbales en se il n'y a pas de choix, l'ajout d'un complément d'agent est généralement impossible. Hamma (2017) signale pourtant qu'avec certaines contraintes sémantiques en français, il est possible d'ajouter un agent.

Les énoncés à un participant soutiennent notre suggestion selon laquelle l'opération sur la valence de type moyen peut servir à mettre en évidence l'évènement en n'insistant ni sur l'agent, ni sur le patient: on peut alors parler de la saillance du procès. Cela se manifeste en particulier dans le cas d'évènements se rapprochant de la voix moyenne, à savoir ceux dont l'unique participant ne peut être considéré ni comme agent, ni comme patient. C'est ainsi que nous arrivons à une sorte d'extrémité d'une échelle, notamment jusqu'au passif résultatif qui, une fois de plus, insiste sur l'évènement et son résultat, sans permettre la référence à l'agent, par exemple la porte est fermée. Ce type de passif est l'unique passif accepté en hongrois : az ajtó be van zárva (« la porte est fermée »).

\section{2. Énoncés avec deux participants}

\subsubsection{Agent et patient}

Le premier ensemble de données contient des énoncés à la forme canonique (ou prototypique ou passif dit achevé) en français, à savoir la construction « être + participe passé » avec un complément d'agent. Ces phrases passives canoniques du français dans lesquelles le sujet est exprimé par un groupe nominal ou par un pronom et le complément d'agent par un groupe prépositionnel ne peuvent être traduites en hongrois que par une phrase transitive active. Le patient reste objet, encodé par l'accusatif. Le hongrois permettant de topicaliser tout constituant, l'objet direct peut facilement se retrouver en tête de phrase. Voici quelques exemples :

(2) 


\begin{tabular}{|l|l|l|l|l|l|}
\hline \multicolumn{6}{|c|}{$j^{\prime}$ 'a été recruté euh sur une petite annonce du Monde par mon/ par mon directeur/ } \\
\hline $\begin{array}{l}\text { engem } \\
\text { 1SG.ACC }\end{array}$ & $\begin{array}{l}\text { egy } \\
\text { IND }\end{array}$ & $\begin{array}{l}\text { apróhirdetésre } \\
\text { petite annonce-SUBL }\end{array}$ & $\begin{array}{l}\text { vett fel } \\
\text { recruter }\end{array}$ & $\begin{array}{l}\text { az } \\
\text { DEF }\end{array}$ & $\begin{array}{l}\text { igazgatóm } \\
\text { directeur-1SG }\end{array}$ \\
\hline
\end{tabular}

\begin{tabular}{|c|c|c|c|c|c|}
\hline \multicolumn{6}{|l|}{ (3) } \\
\hline \multicolumn{6}{|c|}{ je crois que le maire d'Orléans a été en partie trompé par les pouvoirs publics } \\
\hline $\begin{array}{l}\text { azt } \\
\text { DEM-ACC }\end{array}$ & $\begin{array}{l}\text { hiszem, } \\
\text { croire-1SG }\end{array}$ & $\begin{array}{l}\text { hogy az 0-i polgármestert részben } \\
\text { que DEF O.ADJ maire-ACC en partie }\end{array}$ & $\begin{array}{l}\text { becsapták } \\
\text { tromper.PA-3PL }\end{array}$ & $\begin{array}{l}a \\
\mathrm{DEF}\end{array}$ & $\begin{array}{l}\text { hatóságok } \\
\text { pouv.publ. }\end{array}$ \\
\hline & & & & & \\
\hline
\end{tabular}

21 La topicalisation de l'objet en hongrois et du sujet en français peut s'accompagner d'une reprise pronominale :

\begin{tabular}{|c|c|c|c|c|c|}
\hline \multicolumn{6}{|l|}{ (4) } \\
\hline \multicolumn{6}{|c|}{ [un dictionnaire] c'est été ça a été donné par l'école } \\
\hline$a$ & szótárt, & $a z t$ & $a z$ & iskola & adta \\
\hline DEF & dictionnaire-ACC & DEM-ACC & DEF & école & donner-PA-3SG \\
\hline
\end{tabular}

En hongrois moderne, il n'y a pas de construction passive qui correspondrait à celles utilisées dans les langues indo-européennes. La structure des énoncés hongrois ci-dessus peut constituer un argument en faveur de la considération du passif comme la promotion de l'objet direct toutefois du point de vue discursif seulement, par la mise en position topicale en tête de phrase, mais sans sa promotion syntaxique en sujet.

Dans un sous-ensemble de données où il y a toujours un complément d'agent en français, il y a plusieurs traductions possibles en hongrois. La différence entre les traductions peut s'expliquer en partie par le choix du registre, puisque à l'écrit, par exemple dans le journalisme, on peut utiliser une construction avec le participe passé. La phrase (5a) est à la forme active et dans (5b), on trouve un participe passé au sens passif, l'objet restant à l'accusatif.

(5) quand on voit certains certains textes euh qui ont été euh certaines paroles qui ont été dites par des ministres

a. amikor látunk bizonyos szövegeket, bizonyos beszédeket, amiket miniszterek tartottak quand voir-1PL certains texte-PL-ACC certaines discours-PL-ACC REL-PL-ACC ministre-PL tenir-PA3PL

b. amikor látjuk ezeket a miniszterek által tartott beszédeket quand voir-1PL DEM-PL-ACC DEF ministre-PL par tenir-PPA discours-PL-ACC

Dans l'exemple (6a), on trouve une fois de plus la phrase active. Dans (6b), on observe une construction relativement proche d'une forme passive. Rappelons brièvement que la construction formée du verbe d'existence van accompagné du participe adverbial formé avec le suffixe - $v a /-v e^{7}$ est utilisée pour exprimer le passif résultatif, par exemple az ajtó be van zárva 'la porte est fermée'. L'usage de l'autre verbe d'existence, lesz au sens 
original 'devenir', qui peut être utilisé au futur et au passé (lett), est également observable dans le langage courant, mais il est déconseillé par la norme.

Dans l'exemple (6b), le complément d'agent est accompagné de la postposition által 'par' qui était utilisée dans la construction prototypique devenue désuète. Enfin, dans (6c), on trouve une structure considérée comme possible mais plutôt lourde et maladroite qui se compose d'un verbe-support et de la forme nominale, décliné au sublatif. En fait, on entend cette formulation souvent dans des interviews dans lesquels le locuteur vise un style plus soutenu.

(6)

il y a dans le département du Loiret/ un conseiller général communiste/ qui a été élu par les voix radicales

a. van Loiret megyében egy kommunista tanácstag, akit a radikális szavazók választottak meg il y a L. dépt-INESS IND communiste conseiller REL-ACC DEF radical votant-PL élire-PA3PL ACP

b. [...] aki a radikális voksok által lett megválasztva REL DEF radical voix par AUX élire.PADV

c. [...] aki a radikális voksokkal került megválasztásra REL DEF radical voix.INSTR AUX* élection-SUBL

* Le sens de ce verbe est proche de celui de l'angl. get.

\subsubsection{Expérient et stimulus}

Les énoncés qui contiennent un verbe psychologique sont plus bas sur l'échelle de la transitivité (envisagée par Hopper \& Thompson, 1980) puisque l'évènement n'est pas une action qui occasionne un changement d'état chez l'autre participant. Les rôles sémantiques des participants sont expérient et stimulus. L'analyse de quelques exemples permet de percevoir un certain écart par rapport au prototype et de nous approcher vers la notion de saillance du procès.

La structure de la phrase (7) reste proche du prototype, au moins syntaxiquement, puisque c'est une phrase transitive et les deux participants sont des humains : ils se placent donc haut dans la hiérarchie de l'animéité :
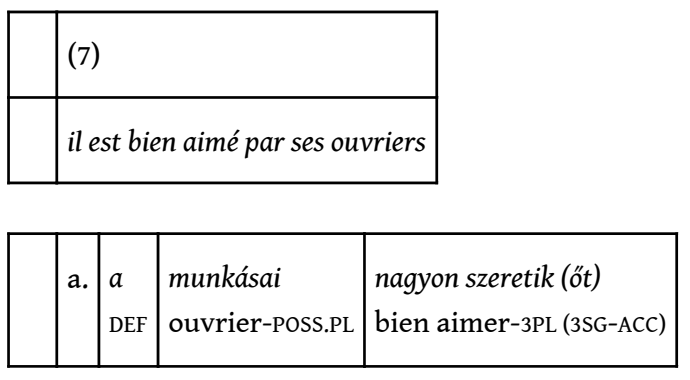

\begin{tabular}{|l|l|l|l|l|l|}
\hline & b. & $\begin{array}{l}\text { szeretik } \\
\text { aimer-3PL }\end{array}$ & $\begin{array}{l}\text { (ót) } \\
\text { (3SG-ACC) }\end{array}$ & $\begin{array}{l}\text { a } \\
\text { DEF }\end{array}$ & $\begin{array}{l}\text { munkásai } \\
\text { ouvrier-POSS.PL }\end{array}$ \\
\hline
\end{tabular}

Dans (7a), la phrase active n'est pas agrammaticale mais comme en français, l'énoncé est au passif, ce n'est certainement pas le constituant ses ouvriers qui devrait occuper la position en tête. L'accent est mis sur l'évènement, non pas sur l'expérient (il), ni sur le stimulus (ses ouvriers). Ainsi, le verbe est focalisé et se trouve en tête de l'énoncé 
puisque le pronom sujet n'apparait pas en hongrois. Le focus est frappé par un accent d'intensité très fort. Tout en insistant sur l'évènement, cette phrase montre aussi que dans certains cas, il n'est pas possible d'éliminer l'agent puisque cela nuirait à l'interprétation soit sémantique soit discursive de la phrase.

Dans la traduction d'autres énoncés contenant des verbes psychologiques, la différence entre les lexiques des deux langues joue un rôle : parfois il y a des adjectifs hongrois qui correspondent exactement aux adjectifs français; dans d'autres cas, il est possible de former le participe adverbial (suffixe - $v a / v e$ ). D'autres exemples montrent que si les verbes psychologiques peuvent apparaitre sous forme d'un verbe moyen intransitif simple, comme megrémül 'prendre peur, être effrayé', dans le langage courant, ils apparaissent aussi comme participes adverbiaux, par exemple meg van hatva 'être ému', meg van döbbenve 'être choqué. Dans les énoncés de ce type, le deuxième participant n'est plus un stimulus mais une cause, exprimée par une subordonnée causale.

Dans les exemples ci-dessous, l'expression de la cause apparait sous forme de subordonnée. Dans (8a), conformément aux règles de la subordination en hongrois, il y a un mot de renvoi, à savoir le pronom démonstratif, décliné ici à l'ablatif. Un nom décliné à l'ablatif pouvait servir de complément d'agent dans le passif canonique aujourd'hui disparu. La traduction de la phrase correspond à l'usage courant ${ }^{8}$.

(8)

Eh bien j'ai été choquée par ce que ce journaliste a dit

\begin{tabular}{|l|l|l|l|l|l|l|l|l|}
\hline & a. & én & $\begin{array}{l}\text { megdöbbentem attól, } \\
1 \mathrm{~S}\end{array}$ & ê. choqué-PA-1SG DEM.ABL & ez & az & újságíró \\
REL-ACC & DEM & DEF & journaliste & dire-PA3SG \\
\hline
\end{tabular}

\begin{tabular}{|l|l|l|l|l|l|l|l|l|l|}
\hline & b. & $\begin{array}{l}\text { hát } \\
\text { eh bien }\end{array}$ & $\begin{array}{l}\text { engem } \\
\text { 1SG.ACC }\end{array}$ & $\begin{array}{l}\text { sokkol, } \\
\text { choquer.3SG }\end{array}$ & $\begin{array}{l}\text { amit } \\
\text { REL.ACC }\end{array}$ & $\begin{array}{l}\text { ez } \\
\text { DEM }\end{array}$ & $\begin{array}{l}\text { az } \\
\text { DEF }\end{array}$ & $\begin{array}{l}\text { újságíró } \\
\text { journaliste }\end{array}$ & $\begin{array}{l}\text { mondott } \\
\text { dire-PA3SG }\end{array}$ \\
\hline
\end{tabular}

\begin{tabular}{|l|l|}
\hline (9) \\
\hline $\begin{array}{l}\text { Ils revinrent tout exaltés } \\
\text { par ce qu'ils avaient vu } \\
\text { et plus encore par ce que leur imagination leur suggérait. }\end{array}$ \\
\hline
\end{tabular}

\begin{tabular}{|l|l|l|l|l|l|l|}
\hline & $\begin{array}{l}\text { Nagyon } \\
\text { très }\end{array}$ & $\begin{array}{l}\text { izgatottan/ } \\
\text { exalté }\end{array}$ & $\begin{array}{l}\text { teljesen } \\
\text { totalement }\end{array}$ & $\begin{array}{l}\text { fellelkesülve } \\
\text { enthousiasmer-PADV }\end{array}$ & $\begin{array}{l}\text { jöttek } \\
\text { venir.PA3PL }\end{array}$ & $\begin{array}{l}\text { vissza } \\
\text { re- }\end{array}$ \\
\hline
\end{tabular}

\begin{tabular}{|l|l|l|l|}
\hline $\begin{array}{l}\text { attól, } \\
\text { DEM.ABL }\end{array}$ & amit & láttak \\
REL-ACC & voir-PA3PL \\
\hline
\end{tabular}




\begin{tabular}{|l|l|l|l|l|l|l|l|}
\hline & és \\
et & $\begin{array}{l}\text { még } \\
\text { encore }\end{array}$ & $\begin{array}{l}\text { inkább attól, } \\
\text { plus DEM.ABL }\end{array}$ & $\begin{array}{l}\text { amit } \\
\text { REL-ACC }\end{array}$ & $\begin{array}{l}\text { a } \\
\text { DEF }\end{array}$ & $\begin{array}{l}\text { képzeletük } \\
\text { imagination.3PL }\end{array}$ & $\begin{array}{l}\text { sugallt. } \\
\text { suggérer-PA3SG }\end{array}$ \\
\hline
\end{tabular}

$(10)$

Desmond Tutu, prix Nobel de la Paix, s'est déclaré choqué par ce qu'il a vu des conditions de vie dans la bande de Gaza.

\begin{tabular}{|l|l|l|l|l|l|l|}
\hline $\begin{array}{l}\text { D.T. } \\
\text { D.T. }\end{array}$ & $\begin{array}{l}\text { béke } \\
\text { paix }\end{array}$ & $\begin{array}{l}\text { Nobel-dijas } \\
\text { Nobel prix-ADJ }\end{array}$ & $\begin{array}{l}\text { azt } \\
\text { DEM-ACC }\end{array}$ & $\begin{array}{l}\text { mondta, } \\
\text { dire-PA.3SG }\end{array}$ & $\begin{array}{l}\text { meg van döbbenve } \\
\text { ê choqué.PADV }\end{array}$ \\
\hline
\end{tabular}

\begin{tabular}{|l|l|l|l|l|l|l|}
\hline $\begin{array}{l}\text { attól, } \\
\text { DEM.ABL. }\end{array}$ & $\begin{array}{l}\text { amilyennek } \\
\text { REL-DAT }\end{array}$ & $\begin{array}{l}\text { az } \\
\text { DEF }\end{array}$ & $\begin{array}{l}\text { életkörülményeket } \\
\text { conditions de vie.PL.ACC }\end{array}$ & $\begin{array}{l}\text { látta } \\
\text { voir-PA.3SG }\end{array}$ & $\begin{array}{l}\text { a G. övezetben } \\
\text { DEF G.bande-INESS }\end{array}$ \\
\hline
\end{tabular}

$(11)$

Moi, j'avais le cœur à l'envers et l'imagination enflammée par ce que je venais d'apprendre sur le sort de Joséphine.

\begin{tabular}{|l|l|l|}
\hline $\begin{array}{l}\text { Nagyon } \\
\text { très }\end{array}$ & $\begin{array}{l}\text { meghatódtam / meg voltam hatva és a } \\
\text { ému-PA.1SG / ê ému.PADV et DEF }\end{array}$ \\
\hline
\end{tabular}

\begin{tabular}{|l|l|l|l|l|l|}
\hline $\begin{array}{l}\text { képzeletem } \\
\text { imagination-1SG }\end{array}$ & $\begin{array}{l}\text { szárnyalt } \\
\text { s'élancer-PA.3SG }\end{array}$ & $\begin{array}{l}\text { attól, } \\
\text { DEM.ABL }\end{array}$ & $\begin{array}{l}\text { amit megtudtam J. } \\
\text { RE-.ACC apprendre-PA.1SG J. }\end{array}$ & $\begin{array}{l}\text { sorsáról. } \\
\text { sort-DEL }\end{array}$ \\
\hline
\end{tabular}

31 L'analyse de la partie du corpus basée sur les verbes psychologiques montre que les subordonnées introduites par par ce que expriment effectivement la cause de l'émotion. Le complément le plus caractéristique d'un verbe moyen est la cause (Keszler, 2000, p. 92), ce qui rend les énoncés ci-dessus faciles à analyser.

32 En guise de conclusion de l'analyse des deux ensembles d'exemples que nous venons d'examiner, on observera les deux énoncés suivants :

\begin{tabular}{|c|c|c|c|c|c|}
\hline \multicolumn{6}{|c|}{ (12) } \\
\hline \multirow[t]{3}{*}{ a. } & \multicolumn{5}{|c|}{ le facteur a été mordu par le chien } \\
\hline & $a$ & kutya & megharapta & $a$ & postást \\
\hline & DEF & chien & mordre-PA.3SG & DEF & facteur-ACC \\
\hline b. & \multicolumn{5}{|c|}{ le facteur a été effrayé par le chien } \\
\hline
\end{tabular}




\begin{tabular}{|l|c|c|l|l|l|l|}
\hline & & a & kutya & megijesztette & $a$ & postást \\
& & DEF & chien & effrayer-PA.3SG & DEF & facteur-ACC \\
& & postás & megijedt & $a$ & kutyától \\
DEF & facteur & prendre peur-PA3SG & DEF & chien.ABL \\
\hline
\end{tabular}

En français dans (12a) et (12b), les structures sont identiques; la traduction permet de voir qu'en hongrois, a. peut être considérée comme canonique et traduite par une phrase active. En revanche, même si la traduction b' par une voix active est également possible, elle est moins naturelle que celle de b", étant donné la hiérarchie entre humain > animé. C'est-à-dire que les énoncés contenant un verbe psychologique sont "moins transitifs", ce qui explique le fait que le locuteur choisira l'énoncé selon ce qu'il souhaite «topicaliser». Cela justifie notre décision de traiter ces énoncés séparément des énoncés passifs prototypiques.

Dans cette première partie, nous avons analysé les énoncés du corpus dans lesquels les deux participants de l'évènement sont explicités. La raison pour laquelle nous considérons ces deux énoncés comme prototypiques est la présence du deuxième participant, sémantiquement agent ou cause. Rappelons que la forme du complément peut être un groupe prépositionnel, un nom décliné ou une subordonnée et dans tous les cas l'énoncé peut être mis en rapport avec un énoncé actif avec un verbe transitif. Cette propriété nous autorise à accepter les analyses en termes de «transformation passive » d'un énoncé transitif, même si la présence de cause à la place d'un agent rend la phrase moins transitive.

Dans la suite, on observera des énoncés sans complément qui, par définition, s'écartent du prototype.

\section{3. Énoncés sans deuxième participant}

La forme des énoncés sans complément d'agent ou sans l'expression d'un autre participant est plus variée dans les deux langues. En français, en dehors de la construction être + participe passé les se-verbes, on trouve le verbe sefaire et les adjectifs en -ble. En hongrois, on trouve le participe adverbial, les adjectifs en -ható et un grand nombre de verbes moyens. Cette variété de formes montre également l'éloignement de ces formes par rapport à la forme canonique.

\subsection{1. être + participe passé}

37 La première solution pour le traducteur reste la phrase active (13a) avec un verbe à la $3^{e}$ personne du pluriel, forme concurrente très fréquente, alors que dans (13b) on observe une nominalisation. Cette construction est correcte et possible, mais elle ne serait pas préférée à l'oral.

(13)

ce qui est contestable c'est la manière dont l'autorité est exercée 


\begin{tabular}{|l|l|l|l|l|l|}
\hline a. & $\begin{array}{l}\text { ami } \\
\text { REL.NOM }\end{array}$ & $\begin{array}{l}\text { vitatható, } \\
\text { contestable }\end{array}$ & $\begin{array}{l}\text { az a mód, ahogy a } \\
\text { DEM DEF manière REL DEF }\end{array}$ & $\begin{array}{l}\text { hatalmat } \\
\text { autorité-ACC }\end{array}$ & $\begin{array}{l}\text { gyakorolják } \\
\text { exercer-3PL }\end{array}$ \\
\hline
\end{tabular}

\begin{tabular}{|l|l|l|l|l|l|}
\hline & b. & $\begin{array}{l}\text { ami } \\
\text { REL.NOM }\end{array}$ & $\begin{array}{l}\text { vitatható, } \\
\text { contestable }\end{array}$ & $\begin{array}{l}\text { az a } \\
\text { DEM DEF }\end{array}$ & $\begin{array}{l}\text { hatalom gyakorlásának módja } \\
\text { autorité exercice.GEN manière-POSS }\end{array}$ \\
\hline
\end{tabular}

Dans (14) et (15), à part la phrase active, on peut utiliser un verbe moyen. Le hongrois connait un grand nombre de paires de verbes « actif - moyen ».

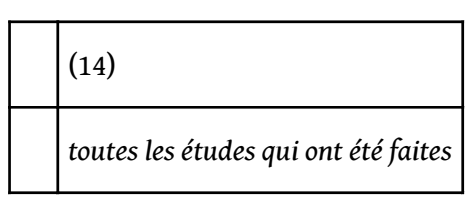

\begin{tabular}{|l|l|l|l|l|}
\hline a. & $\begin{array}{l}\text { minden } \\
\text { tout }\end{array}$ & $\begin{array}{l}\text { tanulmány, } \\
\text { étude }\end{array}$ & $\begin{array}{l}\text { amit } \\
\text { REL.NOM }\end{array}$ & $\begin{array}{l}\text { készítettek } \\
\text { faire.MOY-PA3SG }\end{array}$ \\
\hline
\end{tabular}

\begin{tabular}{|c|c|c|c|c|}
\hline b. & $\begin{array}{l}\text { minden } \\
\text { tout }\end{array}$ & $\begin{array}{l}\text { tanulmány, } \\
\text { étude }\end{array}$ & $\begin{array}{l}a m i \\
\text { REL.NOM }\end{array}$ & $\begin{array}{l}\text { készült } \\
\text { faire.MOY-PA3SG }\end{array}$ \\
\hline
\end{tabular}

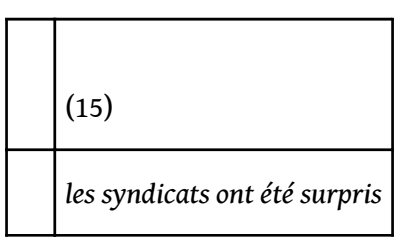

\begin{tabular}{|l|l|l|l|l|}
\hline & a. & $\begin{array}{l}\text { a } \\
\text { DEF }\end{array}$ & $\begin{array}{l}\text { szakszervezetek } \\
\text { syndicat-PL }\end{array}$ & $\begin{array}{l}\text { meglepödtek } \\
\text { s'étonner-PA-3PL }\end{array}$ \\
\hline
\end{tabular}

\begin{tabular}{|l|l|l|l|l|l|l|}
\hline & b. & $\begin{array}{l}\text { a } \\
\text { DEF }\end{array}$ & $\begin{array}{l}\text { szakszervezetek } \\
\text { syndicat-PL }\end{array}$ & $\begin{array}{l}\text { meg } \\
\text { ACP }\end{array}$ & $\begin{array}{l}\text { voltak } \\
\text { etre.PA3PL }\end{array}$ & $\begin{array}{l}\text { lep(öd)ve } \\
\text { s'étonner-PADV }\end{array}$ \\
\hline
\end{tabular}

39 Il y a une différence aspectuelle entre les deux solutions ; b. représente l'accompli par sa forme, mais a. semble plus naturel.

\subsubsection{Verbes « pronominaux»}

40 Parmi les formes non canoniques à valeur passive, les grammaires (par exemple Riegel et coll., 2009, p. 460) reconnaissent traditionnellement les verbes dits «pronominaux ». Il s'agit d'une opération autre que le passif qui sert à diminuer le nombre des arguments d'un verbe. Le résultat est différent, dans la mesure où le rôle sémantique de l'unique argument ne peut être clairement identifié.

41 Notre corpus bilingue contient plusieurs exemples de ce type, même si les constructions sont différentes dans les deux langues observées. Les verbes 
pronominaux du français peuvent être traduits le plus souvent par un verbe moyen en hongrois. Une autre possibilité est l'emploi de la $3^{\mathrm{e}}$ personne du pluriel, une forme concurrente du passif extrêmement fréquente que nous ne traitons pas en détail ici.

Dans (16), en français, on trouve la construction se faire + infinitif qui se traduit ${ }^{9}$ par la $3^{\mathrm{e}}$ personne du pluriel, tout en gardant l'objet-patient à l'accusatif.

\begin{tabular}{|c|c|c|c|}
\hline \multicolumn{4}{|l|}{$(16)$} \\
\hline \multicolumn{4}{|c|}{ se connecte et euh et que d'emblée on se fait euh bombarder de SMS } \\
\hline $\begin{array}{l}\text { aztán } \\
\text { puis }\end{array}$ & $\begin{array}{l}\text { az embert } \\
\text { l'on-ACC }\end{array}$ & $\begin{array}{l}\text { SMS-ekkel } \\
\text { SMS-PL-INSTR }\end{array}$ & $\begin{array}{l}\text { bombázzák } \\
\text { bombarder-3PL }\end{array}$ \\
\hline
\end{tabular}

\begin{tabular}{|l|l|l|}
\hline \multicolumn{3}{|l|}{ et on se fait plus ou moins avoir } \\
\hline $\begin{array}{l}\text { az embert } \\
\text { l'on-ACc }\end{array}$ & $\begin{array}{l}\text { inkább } \\
\text { plutôt }\end{array}$ & $\begin{array}{l}\text { átverik } \\
\text { tromper-3PL }\end{array}$ \\
\hline
\end{tabular}

\subsubsection{Les adjectifs en -ble}

Le dernier ensemble de données se compose des adjectifs en -ble. Il s'agit ici de l'une des possibles lectures d'une forme "moyenne ", appelée facilitative. Dans la plupart des cas, les adjectifs en -ble sont traduisibles par le suffixe -ható (voir ex.13), et la forme négative préfixée par in-/im- l'est par le suffixe -hatatlan. Parfois, l'adjectif de même sens est formé avec un autre suffixe adjectiviseur, par exemple variable 'változó' ou soluble 'oldódó' ; parfois il n'y a pas de traduction exacte, comme introuvable. Pourtant, il n'est pas difficile de faire correspondre le suffixe -ble et le suffixe -ható. Ce dernier est un suffixe complexe qui se compose du suffixe de la potentialité, qui remonte diachroniquement à hat, un verbe dont le sens est 'pouvoir'. Il peut accompagner des verbes transitifs et intransitifs, comme olvas-hat 'lire-POT', fut-hat 'courir-POT', mais le participe adjectival ne peut accompagner qu'un verbe transitif : olvas-hat-ó 'lisible', *futhat-ó. Le rapport avec le passif sera illustré par un exemple :

\begin{tabular}{|l|l|l|}
\hline \multicolumn{2}{|l|}{$(17)$} \\
\hline & a. & On peut lire son écriture \\
b. & son écriture peut être lue \\
c. & son écriture est lisible \\
\hline
\end{tabular}

\begin{tabular}{|l|l|l|l|l|l|}
\hline & a. & $\begin{array}{l}\text { El lehet } \\
\text { ASP possible }\end{array}$ & $\begin{array}{l}\text { olvasni } \\
\text { lire }\end{array}$ & $\begin{array}{l}\text { az } \\
\text { DEF }\end{array}$ & $\begin{array}{l}\text { irását } \\
\text { écriture-POSS-ACC }\end{array}$ \\
\hline b. & --- &
\end{tabular}




\begin{tabular}{|l|l|l|l|l|}
\hline c. & $\begin{array}{l}A z \\
\text { DEF }\end{array}$ & $\begin{array}{l}\text { írása } \\
\text { écriture-POss }\end{array}$ & $\begin{array}{l}\text { olvasható } \\
\text { lisible }\end{array}$ & \\
\hline
\end{tabular}

En l'absence de forme passive canonique, la propriété de l'adjectif attribut qualifie le référent du sujet dans c. qui est l'objet direct dans la phrase active a. Voici encore deux exemples du corpus :

\begin{tabular}{|l|l|l|l|l|l|}
\hline & \multicolumn{3}{|l|}{$(18)$} \\
\hline \multicolumn{4}{|l|}{ Faut dire que mon armoire n'est pas extensible } \\
\hline & $\begin{array}{l}\text { meg kell mondani, } \\
\text { ASP faut dire }\end{array}$ & $\begin{array}{l}\text { hogy a } \\
\text { que DEF }\end{array}$ & $\begin{array}{l}\text { szekrényem } \\
\text { armoire-POSs }\end{array}$ & $\begin{array}{l}\text { nem } \\
\text { NEG }\end{array}$ & $\begin{array}{l}\text { bővíthetó } \\
\text { extensible }\end{array}$ \\
\hline
\end{tabular}

\begin{tabular}{|l|l|l|l|}
\hline & \multicolumn{2}{|l|}{$(19)$} \\
\hline & \multicolumn{2}{|l|}{ c'est encore supportable } \\
\hline & $\begin{array}{l}|l| \\
\text { DEM }\end{array}$ & $\begin{array}{l}\text { még } \\
\text { encore }\end{array}$ & $\begin{array}{l}\text { elviselhetö } \\
\text { supportable }\end{array}$ \\
\hline
\end{tabular}

L'emploi de ce suffixe peut donc être rangé parmi les formes non canoniques du passif, aussi bien en français qu'en hongrois.

\subsubsection{Formes concurrentes et autres remarques}

Toutes les formes concurrentes n'ont pas été analysées dans le présent travail ; leur exploitation du point de vue proposé ici reste une perspective intéressante. Nous en mentionnons quelques-unes.

Dans plusieurs langues (Keenan \& Dryer, 1981, p. 4 mentionnent le kru et l'hébreu), la forme concurrente la plus fréquente est la $3^{\mathrm{e}}$ personne du pluriel impersonnel. Les exemples (13) et (14) en hongrois représentent cette solution. En outre, en hongrois, on rencontre une locution périphrastique avec une nominalisation, voir ex. (6c), dans laquelle l'objet-patient devient sujet comme dans un « vrai » passif, mais cette tournure est considérée comme compliquée et lourde. Pour terminer, il faut mentionner que la construction hongroise "verbe d'état + participe adverbial " remplit dans tous les registres le rôle du passif disparu pour exprimer le résultat d'une action alors que pour exprimer le procès, son emploi est déconseillé : a munka be van fejezve 'le travail est terminé' vs a munka be lett fejezve 'le travail a été terminé'.

\section{Conclusions}

Nous avons traité deux ensembles de données que l'on peut appeler respectivement "passif canonique» et "non canonique». Le critère fondamental pour établir la distinction entre canonique ou non n'était pas la forme de la construction, à savoir la 
forme verbale "être + participe passé » du français, mais la présence ou non du deuxième participant de l'action.

Dans un premier sous-ensemble, il y a des énoncés transitifs dans lesquels les rôles sémantiques des deux participants sont respectivement agent et patient. Après l'observation des traductions du corpus, on constate qu'en hongrois, dans ces énoncés actifs, l'ordre des constituants varie selon les rôles pragmatiques, ce qui permet de topicaliser l'objet direct en le mettant en tête de phrase, sans recourir à une construction passive. Le premier ensemble de données examiné contient ainsi des énoncés ayant la forme canonique du passif en français, avec complément d'agent.

Dans le deuxième sous-ensemble se trouvent des énoncés moins transitifs qui contiennent un verbe psychologique et dans lesquels les rôles sémantiques sont expérient et stimulus. La forme des énoncés français reste canonique, alors que le deuxième participant, à savoir le stimulus peut être exprimé par une subordonnée causale. Les énoncés hongrois qui correspondent aux énoncés français peuvent prendre la forme d'une phrase active, mais on utilise le plus souvent un verbe moyen et le deuxième participant est exprimé soit par une subordonnée causale, soit par un nom décliné à l'ablatif.

51 Le second ensemble de données contient des formes considérées non canoniques en français, les $s e$-verbes et les adjectifs en -ble, auxquelles peuvent correspondre en hongrois des verbes moyens et des adjectifs en -ható/-hetö. Dans l'écrasante majorité des cas, ces énoncés n'acceptent pas l'introduction du deuxième participant, dans aucune des langues.

Quoique toutes les formes concurrentes n'aient pas été étudiées ici, notre hypothèse sur la distinction entre passif canonique et non canonique se voit corroborée par l'étude du corpus. Nous avons considéré la présence ou l'absence de l'expression de l'agent comme critère définitoire. Ainsi, il est possible de délimiter le véritable passif qui relève de la diminution de l'importance de l'agent et l'ensemble d'autres phénomènes plus proches du passif, à savoir les énoncés qui expriment la cause de l'évènement, ainsi qu'un dernier sous-ensemble, celui des énoncés qui s'approchent $\mathrm{du}$ moyen et qui n'autorisent pas l'introduction du deuxième participant. En dehors du premier ensemble de données avec la présence de l'agent, les autres, s'approchant du moyen, permettent de mettre l'accent sur l'évènement même, c'est pourquoi nous suggérons de parler de la « saillance » du procès.

\section{BIBLIOGRAPHIE}

BENVENISTE, Émile. (1966). Problèmes de linguistique générale. Gallimard.

BRAHIM, Ahmed. (2001). Passif et moyen dans les langues du pourtour méditerranéen. Linx, 45, 107-116.

CREISSELS, Denis. (2001). Remarques sur la notion de passif et l'origine des constructions passives. Linx, $45,71-82$. 
CREISSELS, Denis. (2006). Syntaxe générale. Une introduction typologique. Lavoisier.

HAMmA, Badreddine. (2017). Tentative de classification des « compléments d'agent » dans les phrases passives achevées et dans les énoncés longs à sens passif. ELA, 187(3), 311-324.

HAMмA, Badreddine. (2019). Quand l'interaction n'est pas là, la souris est mangée par le chat ! Remarques sur l'enseignement du passif en classe de français. Dans A.-S. Calinon, B. Hamma, K. Ploog \& M. Skrovec, Linguistique interactionnelle, grammaire de l'oral et didactique du français (p. 237-262). Presses universitaires de Franche-Comté.

HAMMA, Badreddine. (2020). Pourquoi ne peut-on pas se passer de «l'agent passif» dans une vraie conversation ? Dans J. Chaker, L. Oueslati \& L. Hosni, Le dialogue et la conversation à la croisée des approches (p. 87-103). Latrach Édition.

HAMMA, Badreddine, TARDIF, Amélie \& BADIN, Flora. (2017). Le passif à l'oral. Fiche FRACOV :

$<$ www.ortolang.fr/market/corpora/fracov?path=\%2F>.

HoPPeR, Paul J. \& THOMPson, Sandra A. (1980) Transitivity in Grammar and Discourse. Language, 56(2), 251-299.

KeENAN, Edward L. \& DRYeR, Matthew S. (1981). Passives in the World's Languages. Dans T. Shopen (dir.), Language Typology and Syntactic Description (vol. I, p. 325-361). Cambridge University Press.

KeMmer, Suzanne. (1993). The Middle Voice. John Benjamins.

LYONS, John. (1970). Linguistique générale. Larousse.

KESZLER, Borbála (dir.). (2000). Magyar grammatika [Grammaire du hongrois]. Nemzeti Tankönyvkiadó.

RIEGEL, Martin, Pellat Jean-Christophe \& Rioul, René. (2009). Grammaire méthodique du français ( 4 e éd.). Presses universitaires de France.

SHIBATANI, Masayoshi. (1985). Passive and Related Constructions: A Prototype Analysis. Language, 61(4), 821-848.

SőRÉs, Anna. (2003). À propos du passif en français et en hongrois. Dans T. Szende \& G. Máté (dir.), Frontières et passages. Actes du colloque franco-hongrois sur la traduction (p. 265-274). Peter Lang.

SŐRÉs, Anna. (2006). Le hongrois dans la typologie des langues. Lambert \& Lucas.

SőRÉs, Anna. (2013). Middle Verb Morphology in Hungarian and in a Cross-Linguistic View. Dans Actes du Congrès international des linguistes. <www.cil19.org/uploads/documents/ Middle_verb_morphology_in_Hungarian_and_in_a_cross-linguistic_view.pdf>. Source des exemples de l'oral : ESLO (Enquête sociolinguistique à Orléans), <http://eslo.humanum.fr>.

\title{
ANNEXES
}

\section{Abréviations}

\author{
$\mathrm{ABL}=$ ablatif \\ $\mathrm{ACC}=$ accusatif \\ $\mathrm{ACP}=$ aspect accompli
}




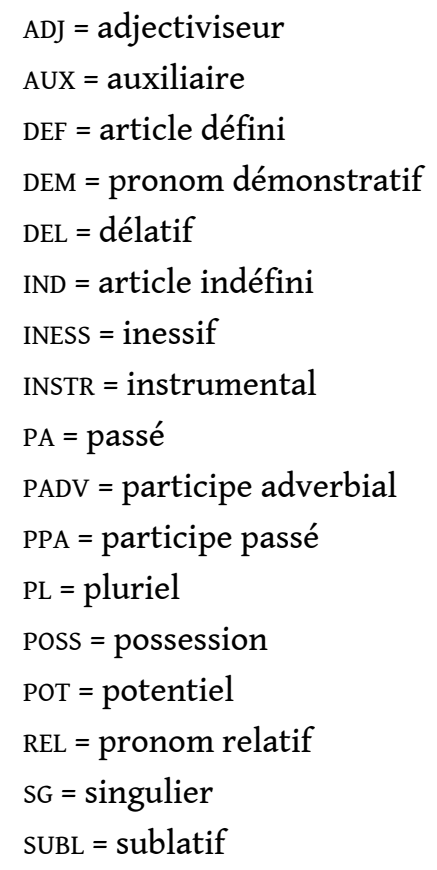

\section{NOTES}

1. Le morphème passif du hongrois a disparu de l'usage au tout début $d u x^{e}$ siècle. La langue d'aujourd'hui se sert de plusieurs constructions périphrastiques dont les plus importantes sont décrites au point 3 .

2. Là où c'est nécessaire, nous nous servons aussi de la terminologie sémantique en évoquant un évènement et un certain nombre de participants, ces derniers étant encodés syntaxiquement comme sujet, objet, etc.

3. Le passif impersonnel du type il a été décidé que existe en français aussi, mais il ne sera pas traité dans cette étude.

4. Le sujet est " affecté » par opposition à l'objet direct/patient qui subit un changement d'état (Creissels, 2006, I, p. 281).

5. Par exemple, Riegel et coll. (2009), 437 établissent le lien entre passif et moyen mais optent pour le maintien du terme «tournure pronominale».

6. Les énoncés en hongrois ne sont pas segmentés en morphèmes car pour des raisons diachroniques, la segmentation du radical et des affixes est souvent problématique. Dans les gloses, les tirets permettent de voir l'ordre et la nature des morphèmes. Le point signale un morphème porte-manteau.

7. Allomorphie du suffixe permettant de respecter les règles de l'harmonie vocalique.

8. Les exemples de 8 à 11 sont empruntés au corpus de Hamma (2017, 2019). Il contient des énoncés pris non seulement de sources orales comme ESLO mais de Frantext et de la presse écrite également, ce qui explique la présence d'un passé simple.

9. Le hongrois ne connait pas de pronom personnel équivalant à « on », az ember 'l'homme' doit être inséré. 


\section{RÉSUMÉS}

Cette étude propose l'hypothèse que les formes dites «non canoniques » du passif, notamment les énoncés sans complément d'agent ou de cause se rapprochent davantage de la voix moyenne que du passif. L'intérêt de l'étude contrastive est le fait qu'en hongrois moderne, il n'y a pas de construction passive correspondant à la forme canonique du français. Ce type de phrase est traduit par une phrase active, avec topicalisation de l'objet direct.

Après un bref survol des notions de base telles que la détransitivisation et la voix moyenne, nous étudions un ensemble d'énoncés oraux en français et leur traduction en hongrois. L'analyse se focalise sur les formes non canoniques dans les deux langues, respectivement, les se-verbes et les adjectifs en -ble, et les verbes moyens et des adjectifs en -ható/-hetö. Les observations selon lesquelles les formes non canoniques ne permettent pas l'ajout d'un complément corroborent également l'idée que ces constructions, en écartant l'agent ou la cause, soulignent le procès et son résultat.

The paper proposes the hypothesis that "non-canonical passives", namely sentences without agent or cause are closer to middle voice than to passive voice. The point of the contrastive study is that Modern Hungarian has no passive construction similar to French canonical passives. A passive sentence in French can only be translated in Hungarian by an active sentence with a topicalized direct object.

After a brief overview of some basic concepts such as detransitivisation and middle voice, a set of French passives translated in Hungarian are studied. The analysis focuses on non-canonical passives in both languages, namely se-verbs and adjectives suffixed by -ble in French, middle verbs and adjectives suffixed by -ható/-hetö in Hungarian. We observe that non-canonical forms do not allow agent or cause complements and so they corroborate the idea that these constructions removing agent or cause can emphasize the event and its result.

\section{INDEX}

Mots-clés : passif non canonique, analyse contrastive, topicalisation, agent, voix moyenne

Keywords : non-canonical passive, contrastive analysis, topicalization, agent, middle voice

\section{AUTEUR}

\section{ANNA SŐRÉS}

Université Lumière Lyon 2, Laboratoire DDL

anna.sores@univ-lyon2.fr 